\title{
Anarchy and Neutrino Physics
}

\section{Luc Marleau*t, Jean-François Fortin and Nicolas Giasson}

Département de physique, de génie physique et d'optique, Université Laval, Québec, Canada

E-mails: lmarleau@phy.ulaval.ca

jean-francois.fortin@phy.ulaval.ca

nicolas.giasson.1@ulaval.ca

\begin{abstract}
The anarchy principle leading to the seesaw ensemble is studied analytically with the usual tools of random matrix theory. The probability density function for the seesaw ensemble of $N \times N$ matrices is obtained in terms of a multidimensional integral (where $N$ corresponds to the number of generations). This probability density functions is then used to extract information on the relevant physical parameters of the neutrino sector of a seesaw-extended Standard Model. For $N=3$, a probability test is introduce to help characterize the type I-III and type II seesaw ensembles using numerical integration methods. A systematic comparison between the two ensembles is performed to point out the fundamental differences between them. It is found that the type I-III seesaw ensemble is better suited to accommodate experimental data. Moreover, the results indicate a strong preference for the mass splitting associated to normal hierarchy. However, because of the decoupling of the probability density function for the light neutrino masses and the neutrino mixing angles and phases, all permutations of the singular values are found to be equally probable for a particular mass splitting, which implies that predictions regarding the hierarchy of the mass spectrum remains out of reach in this particular framework.
\end{abstract}

EPS-HEP 2017, European Physical Society conference on High Energy Physics

5-12 July 2017

Venice, Italy

\footnotetext{
* Speaker.

${ }^{\dagger}$ Work supported by the NSERC (Canada).
} 


\section{Introduction}

Neutrinos are only sensitive to weak interactions and gravity. Therefore, to accurately describe their interactions one only needs left-handed Weyl spinors. The Standard Model (SM), being as economic as possible in its particle content, does not incorporate right-handed neutrinos since they are not required in any known gauge interactions (and there is no experimental evidence of their existence). The highly constrained particle content of the SM, combined with gauge symmetry, Lorentz invariance and the requirements of a renormalisable theory, leads to the conclusion that neutrinos are massless in the SM. However, experiments on neutrino oscillations have provided compelling arguments in favor of non-zero masses for the three generations and have actively contributed in revealing the irregular nature of the neutrino sector. In an effort to explain the peculiarities of the neutrino sector by exploiting this lack of discernible structure or flavor symmetry, the anarchy principle was introduced in [1]. Relying on extensions of the SM based on the three seesaw mechanisms, this hypothesis consist in postulating that the low-energy neutrino mass matrix generated by one of the seesaw mechanisms is obtained from randomly-generated high-energy mass matrices with elements distributed following Gaussian-like ensembles. This brief communication presents some of the most recent results obtained by adopting the anarchy hypothesis as well as its consequences for low energy neutrino physics.

\section{Seesaw mechanisms}

There exist three basic seesaw mechanisms which can be used to extend the SM in the neutrino sector. They require modifying the particle content of the SM by adding (hypothetical) heavy degrees of freedom so that, for example, couplings of the form $\mathscr{L} \propto-Y \bar{L} \Phi_{c} v_{R}-\frac{1}{2} \overline{v_{R}^{c}} M^{R} v_{R}+$ h.c can be generated, leading to a low energy mass matrix $M^{v}$ for the light neutrinos. Here, $L$ is a left-handed lepton doublet, $\Phi$ is the Higgs doublet and $v_{R}$ is a heavy right-handed neutrino singlet.

\subsection{Type I, II and III}

In the type I seesaw mechanism, three heavy right-handed neutrinos singlets are added to the particle content. In the type II seesaw mechanism, a heavy scalar Higgs triplet $\Delta$ is added to the particle content. Finally, in the type III seesaw mechanism, three heavy fermionic triplets $\Sigma$ are added to the particle content. The resulting mass matrices are

$$
M^{v}= \begin{cases}-M^{D}\left(M^{R}\right)^{-1}\left(M^{D}\right)^{T}, & \text { Type I } \\ M^{L}, & \text { Type II } \\ -M^{D}\left(M^{\Sigma}\right)^{-1}\left(M^{D}\right)^{T}, & \text { Type III }\end{cases}
$$

where $M^{R}, M^{L}$ and $M^{\Sigma}$ are the symmetric Majorana mass matrices of the right-handed neutrinos, left-handed neutrinos and the right-handed fermionic triplets respectively. Naturally, $M^{L}$ is related to the mass of the heavy Higgs triplet according to $M^{L}=v^{2} \lambda_{\Delta} Y^{\Delta}\left(M^{\Delta}\right)^{-1}$. Concerning the Dirac mass matrices of the type I and type III seesaw mechanism, they are defined as $M^{D} \equiv \frac{v}{\sqrt{2}} Y^{v}$ for the type I and $M^{D} \equiv \frac{v}{\sqrt{2}} Y^{\Sigma}$ for the type III. Here, $v$ is the Higgs VEV and $Y^{v}, Y^{\Delta}$ and $Y^{\Sigma}$ are the Yukawa-like couplings of the newly introduced particles. Thus, these seesaw mechanisms 
- can predict small neutrino masses for the three active neutrinos;

- are based on hybrid mass terms of Majorana type;

- cannot explain or predict the various couplings and parameters that are contained in the mass matrices $M^{D}, M^{R}, M^{\Sigma}$, etc.

It should be noted that the type III seesaw mechanism, although having a different origin, produces a mass matrix with the same structure as the one in type I for the light neutrinos. These two seesaw scenarios are therefore undistinguishable in the framework of anarchy.

\section{Probability density function}

In order to overcome the lack of knowledge on the underlying theory of neutrino masses, the anarchy scenario proposed by Murayama [1] is assumed. Therefore, the probability density function (pdf) for neutrino masses and mixings can be derived analytically from first principles for each seesaw scenario, an idea that was first alluded to in [2]. First, we need to adopt a particular framework that allows us to obtain quantitative results with the tools develop in random matrix theory (RMT). This leads to the following three hypotheses. (i) There is no physical distinction among the three generations of lepton doublets (they have the same interactions). (ii) The various couplings and mass matrix elements are randomly generated from a distribution which is basis independent. (iii) The matrix elements are distributed independently from each other. These specific constraints uniquely determine the joint probability density function $\mathscr{P}$ (jpdf) for the matrix elements with which we scan the parameter space. This is a well-known result in RMT and it corresponds to the gaussian distribution. For example

$$
\begin{aligned}
& \mathscr{P}_{D}\left(M^{D}\right) d M^{D}=\left(\frac{1}{2 \pi \Lambda_{D}^{2}}\right)^{N^{2}} e^{-\frac{1}{2 \Lambda_{D}^{2}} \operatorname{tr}\left(\left(M^{D}\right)^{\dagger} M^{D}\right)} \prod_{i, j}^{N} d M_{i j}^{D} \\
& \mathscr{P}_{A}\left(M^{A}\right) d M^{A}=\left(\frac{1}{2}\right)^{N}\left(\frac{1}{\pi \Lambda_{A}^{2}}\right)^{\frac{N(N+1)}{2}} e^{-\frac{1}{2 \Lambda_{A}} \operatorname{tr}\left(\left(M^{A}\right)^{\dagger} M^{A}\right)} \prod_{i \leq j}^{N} d M_{i j}^{A},
\end{aligned}
$$

where the superscript $A=R, L, \Sigma$ identifies the corresponding Majorana matrix. Then, we wish to compute $\mathscr{P}_{v}\left(M^{v}\right) d M^{v}$ for each seesaw scenario. Following the framework previously described, this quantity characterizes specific random matrix ensembles, known as the seesaw ensembles.

\subsection{Type I-III seesaw ensemble}

Consider, for example, the type I mass matrix. The resulting distribution can be obtained by following a few steps. Step 1: Write down the joint density for $M^{D}$ and $M^{R}$ assuming that they are statistically independent. Step 2: Introduce a $M^{v}$ dependence by an appropriate change of variables $\left(M^{R}=\left(M^{D}\right)^{T}\left(M^{v}\right)^{-1} M^{D}\right)$. This gives us a joint density for $M^{v}$ and $M^{D}$. Step 3: Compute the associated Jacobian $|\operatorname{det} J|$ and introduce the dimensionless quantity $M^{v}=\hat{M}^{v} /\left(\sqrt{2} \Lambda_{v}\right)$, where $\Lambda_{v}$ is the light neutrino mass scale. Step 4: Obtain $\mathscr{P}_{V}\left(\hat{M}^{v}\right)$ by marginalizing (integrating) out $M^{D}$. This step is easier to achieve when the pdf is written in terms of the singular values of each matrix, leading a complicated integral form [3].

$$
\underbrace{\mathscr{P}_{v}^{\mathrm{I}-\mathrm{III}}\left(\hat{m}_{1}^{v}, \ldots, \hat{m}_{N}^{v}\right) d \hat{m}_{1}^{v} \ldots d \hat{m}_{N}^{v}}_{\text {singular values }} \propto I_{N}\left(\hat{m}_{1}^{v}, \ldots, \hat{m}_{N}^{v}\right) \prod_{i<j}^{N}\left|\left(\hat{m}_{i}^{v}\right)^{2}-\left(\hat{m}_{j}^{v}\right)^{2}\right| \prod_{k=1}^{N}\left|\hat{m}_{k}^{v}\right|^{-(2 N+1)} d \hat{m}_{k}^{v}
$$


where

$$
\begin{aligned}
& I_{N}\left(\hat{m}_{1}^{v}, \ldots, \hat{m}_{N}^{v}\right)=\int_{U \in \mathscr{V}_{N}} \int_{0}^{\infty} \ldots \int_{0}^{\infty} \prod_{i<j}^{N}\left|x_{i}-x_{j}\right|^{2} e^{-2\left|\sum_{k=1}^{N} \frac{U_{k i} L_{k j}}{\hat{m}_{k}^{v}}\right|^{2} x_{i} x_{j}} \times \\
& \prod_{i=1}^{N} x_{i}^{N+1} e^{-x_{i}\left(1+\left|\sum_{k=1}^{N} \frac{U_{k i}^{2}}{\tilde{m}_{k}^{k}}\right|^{2} x_{i}\right)} d x_{i} \frac{(2 \pi)^{N}\left(U^{\dagger} d U\right)^{\prime}}{\operatorname{Vol}\left(\mathscr{V}_{N}\right)} .
\end{aligned}
$$

\subsection{Type II seesaw ensemble}

Consider the type II mass matrix. In this case, the resulting pdf is much simpler since the symmetric mass matrix $M^{L}$ is directly distributed according to $\mathscr{P}_{L}\left(M^{L}\right) d M^{L}$ (see (3.1)). Then, to extract information on the relevant physical parameters, one needs only to express this pdf in terms of its singular values.

$$
\mathscr{P}_{v}^{\mathrm{II}}\left(\hat{m}_{1}^{v}, \ldots, \hat{m}_{N}^{v}\right) d \hat{m}_{1}^{v} \ldots d \hat{m}_{N}^{v} \propto \prod_{i<j}\left|\left(\hat{m}_{i}^{v}\right)^{2}-\left(\hat{m}_{j}^{v}\right)^{2}\right| \prod_{i=1}^{N}\left|\hat{m}_{i}^{v}\right| e^{-\left(\hat{m}_{i}^{v}\right)^{2}} d \hat{m}_{i}^{v} .
$$

\section{The case $N=3:$ SM neutrino physics}

Integrals of the previous section can be computed numerically (with the use of an adaptive Monte Carlo algorithm) to obtain the desired marginal pdfs for the neutrino masses [4]. Moreover, to help quantify the underlying trend in both ensembles, the following probability test is introduced [4].
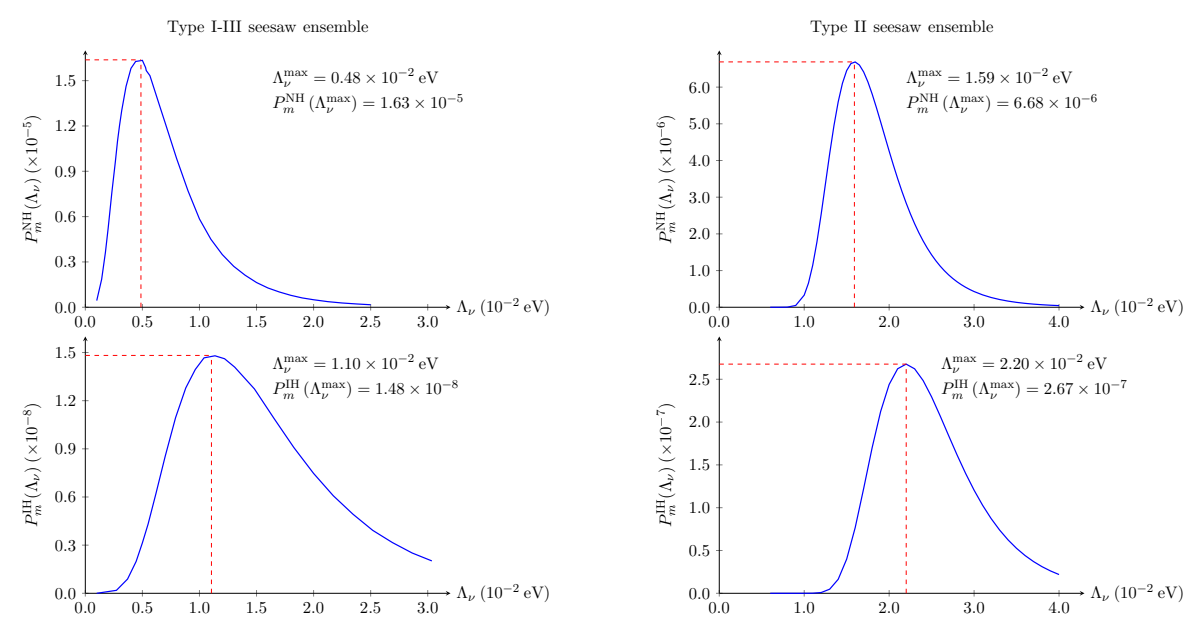

This probability test implies that the pdfs for the seesaw ensembles are integrated over the allowed region defined by experimental data at $1 \sigma$ for normal $(\mathrm{NH})$ and inverted (IH) hierarchy $[5,6]$. Since the only free parameter left in the pdfs is $\Lambda_{v}$, the idea is to plot the probability as a function of $\Lambda_{v}$ over a range were the curves reach a maximum. This allows for a simple comparison of their maximum values (by taking appropriate ratios) to determine the likelihood of each ensemble to generate the observed values. 


\section{Discussion and conclusion}

From the study of the pdfs of the seesaw ensembles and the probability test, we can deduce some important properties of the neutrino sector [3, 4]. First, since these pdfs are invariant under permutations of the singular values $\hat{m}_{1}, \hat{m}_{2}, \hat{m}_{3}$, it means that all permutations of the singular values are equally probable for a particular mass splitting, yielding a lack of predictive power regarding the neutrino mass hierarchy. Moreover, by studying the asymptotic behaviors of the mass distribution, it is possible to deduce that the seesaw ensembles favor three massive light neutrinos, a massless neutrino is forbidden [3]. From the probability test one can see that the type I-III seesaw ensemble is $\sim 1000$ times more likely to generate parameters that are contained in the region defined by the normal hierarchy data set than in the inverted hierarchy data set. For the type II seesaw ensemble, the same tendency is observed but in smaller proportion (about $\sim 25$ times more likely). Therefore, the probability test can be used as a selection tool to identify which ensemble is better suited to generate parameters within the region defined by normal hierarchy. It is found that the type I-III is $\sim 2$ times better than the type II seesaw ensemble. Finally, for all seesaw mechanisms, the preferred neutrino energy scale is $\Lambda_{v} \sim \mathscr{O}\left(10^{-2}\right) \mathrm{eV}$, which leads to a scale of new physics similar to the GUT scale when the associated coupling constants are of order one. Smaller coupling constants can partly reduce the new physics scale.

\section{References}

[1] L. J. Hall, H. Murayama and N. Weiner, Neutrino mass anarchy, Phys. Rev. Lett. 84 (2000) 2572-2575, [hep-ph/9911341].

[2] Y. Bai and G. Torroba, Large N (=3) Neutrinos and Random Matrix Theory, JHEP 12 (2012) 026, [1210.2394].

[3] J.-F. Fortin, N. Giasson and L. Marleau, Probability density function for neutrino masses and mixings, Phys. Rev. D94 (2016) 115004, [1609.08581].

[4] J.-F. Fortin, N. Giasson and L. Marleau, Anarchy and Neutrino Physics, JHEP 04 (2017) 131, [1702.07273].

[5] G. Pagliaroli, F. Rossi-Torres and F. Vissani, Neutrino mass bound in the standard scenario for supernova electronic antineutrino emission, Astropart. Phys. 33 (2010) 287-291, [1002 . 3349].

[6] M. C. Gonzalez-Garcia, M. Maltoni and T. Schwetz, Updated fit to three neutrino mixing: status of leptonic CP violation, JHEP 11 (2014) 052, [1409.5439]. 